\title{
FIELDS OF EXTREMALS IN SPACE
}

\author{
BY \\ GILBERT AMES BLISS AND MAX MASON*
}

The minimizing space curves for the integral

$$
J=\int F\left(x, y, z, x^{\prime}, y^{\prime}, z^{\prime}\right) d t
$$

form a family which contains four arbitrary constants. Of special interest are the two-parameter families which pass through a given point or are cut transversally by a given curve or surface. Such families occur when the integral $J$ is to be minimized with respect to curves which join two fixed points or which have one end-point fixed while the other is free to vary on a fixed curve or surface. $\dagger$ In the study of the sufficient conditions which insure a minimum for the integral $J$ under these conditions it is essential to know that such two-parameter families fill out simply a portion of space about the point through which all of the curves of the family pass, or about the curve or surface by which they are cut transversally. The minimizing curves, the extremals, are said to form a field in the region in which this property holds.

The field proofs for the analogous cases in the plane have already been made. $\neq$ The proof given by BoLzA for the existence in the plane of a field about an arc of an extremal which does not contain a pair of conjugate points, can be extended readily to the analogous case in space. This proof includes the field proof mentioned above for the extremals which are cut transversally by a given surface.

It is to be shown here that the extremals through a fixed point $\S$ or the extremals to which a given curve is transversal also form fields. In these two cases the functional determinant of the equations to be solved vanishes at the point or along the curve in question. For the former case a suitably selected transformation of the variables reduces the equations to a set whose functional determinant does not vanish. The solution is then obtained from the theory of

* Presented to the Society, Sept. 1, 1908.

† These minimizing problems were considered by the writers in a paper entitled "The properties of curves in space which minimize a definite integral," vol. 9 (1908), p. 440.

† See BolzA, Vorlesungen über Variationsrechnung, pp. 164, 249, 271.

8 The construction of a field formed by extremals through a fixed point has been previously disoussed by two writers, both of whom used methods quite different from the one given here. See Bill, The construction of a space field of extremals, Bulletin of the A merioan Mathematical Society, vol. 15 (1909), p. 374 ; also HADAMARD, Leģons sur le calcul des variations, Note A, p. 497. 
implicit functions by the aid of a theorem due to BoLzA, ${ }^{*}$ which is proved in $\S 1$.

A different method is used in $\S 3$ to prove the existence of a field about a curve $L$, but either this or the method of $\S 2$ is applicable to both problems. In order to apply the method of $\S 2$ to prove the existence of a field about $L$ it is necessary first to construct a system of polar coördinates in the neighborhood of $L$ with properties similar to those of the well-known ones used in $\S 2$.

\section{$\S 1$. Bolza's theorem concerning implicit functions.}

Consider a set of equations

$$
f_{i}\left(x_{1}, x_{2}, \ldots, x_{m}: y_{1}, y_{2}, \cdots, y_{n}\right)=0 \quad(i=1,2, \cdots, n),
$$

for which the functions $f_{i}$ satisfy the following conditions :

$A$ ) The functions $f_{i}$ are continous and have continuous first partial derivatives with respect to the $y$ 's for all values $\left(x_{1}, x_{2}, \ldots, x_{m}: y_{1}, y_{2}, \ldots, y_{n}\right)$ in a certain region $\mathfrak{A}$.

$B)$ There exists a finite closed set $\mathfrak{C}$ of solutions $\left(x_{1}, x_{2}, \ldots, x_{m}: y_{1}, y_{2}, \ldots, y_{n}\right)$ of equation (1) entirely within the region $\mathfrak{A}$. The projection of $\mathfrak{C}$ in the $\left(x_{1}, x_{2}, \ldots, x_{m}\right)$-space will be denoted by $\mathfrak{h}$.

$C)$ No two distinct points in $\mathfrak{C}$ have the same projection in $\mathfrak{H}$ : in other words, to any point $\left(x_{1}, x_{2}, \ldots, x_{m}\right)$ in $\mathfrak{S}$ there corresponds one and but one solution $\left(x_{1}, x_{2}, \ldots, x_{m}: y_{1}, y_{2}, \ldots, y_{n}\right)$ in $\mathfrak{C}$.

$D$ ) In the set $\mathfrak{C}$ the functional determinant $\partial\left(f_{1}, f_{2}, \ldots, f_{n}\right) / \partial\left(y_{1}, y_{2}, \ldots, y_{n}\right)$ is always different from zero.

Then there exists a constant $\epsilon$ such that no two solutions of equations (1) in $\mathfrak{E}_{\epsilon} \dagger$ possess the same projection in $\mathfrak{W}_{\epsilon}$, and a constant $\delta \leqq \epsilon$ can be determined so that to any point in the neighborhood $\mathfrak{H}_{\delta}$ there corresponds at least one solution of equations $(1)$ in the neighborhood $\mathfrak{E}_{\epsilon}$. The functions

$$
y_{1}\left(x_{1}, x_{2}, \ldots, x_{m}\right), y_{2}\left(x_{1}, x_{2}, \ldots, x_{m}\right), \ldots, y_{n}\left(x_{1}, x_{2}, \ldots, x_{m}\right)
$$

so defined in $\mathfrak{K}_{\delta}$ are of class $C$, and if the function $f_{i}$ are of class $C^{(n)} \ddagger$ in $\mathfrak{A}$ these functions are also of class $C^{(n)}$ in $\mathfrak{W}_{\delta}$.

* Loc. cit., p. 166. BoLza makes the proof of the general theorem depend upon a special case which he has discussed in the preceding pages. The theorem was proved independently by the writers for the purposes of the present paper, and the proof in $\$ 1$ is the direct proof which they derived, the statement of the theorem being slightly different from that of Bolza.

†See Bolza, loc. cit., p. 154. His notation is $(\varepsilon)_{\mathfrak{c}}$. The neighborhood $\mathfrak{E}_{e}$ for any set $\mathfrak{E}$ and any constant $\varepsilon$ is the set of points $\left(x_{1}, x_{2}, \cdots, x_{m}: y_{1}, y_{2}, \cdots, y_{n}\right)$ for each of which there exists at least one point $\left(x_{1}{ }^{\prime}, x_{2}{ }^{\prime}, \cdots, x_{m^{\prime}}{ }^{\prime}: y_{1}{ }^{\prime}, y_{2}{ }^{\prime}, \cdots, y_{n^{\prime}}\right)$ in $\mathbb{E}$ satisfying the relations

$$
\left|x_{1}-x_{1}^{\prime}\right|<\varepsilon,\left|x_{2}-x_{2}^{\prime}\right|<\varepsilon, \cdots,\left|x_{m}-x_{m^{\prime}}\right|<\varepsilon,
$$

with similar inequalities for $y_{1}, y_{2}, \cdots, y_{n}$.

$\ddagger$ A function is of class $C^{(n)}$ if its derivatives up to and including those of order $n$ are continuous. 
As a first step in the proof it can be shown that a constant $\epsilon$ exists such that no two distinct solutions of equations (1) in the neighborhood $\mathfrak{E}_{e}$ can have the same projection $\left(x_{1}, x_{2}, \ldots, x_{m}\right)$ in $\mathfrak{S}_{e}$. For if this were not the case two sequences of solutions of equations (1)*

$$
\begin{gathered}
\left\{x_{1}^{(i)}, x_{2}^{(i)}, \ldots, x_{m}^{(i)}: y_{1}^{(i)}, y_{2}^{(i)}, \cdots, y_{n}^{(i)}\right\}, \\
\quad\left\{x_{1}^{(i)}, x_{2}^{(i)}, \ldots, x_{m}^{(i)}: Y_{1}^{(i)}, Y_{2}^{(i)}, \cdots, Y_{n}^{(i)}\right\} \\
\left(y_{1}^{(i)}, y_{2}^{(i)}, \cdots, y_{n}^{(i)}\right) \neq\left(Y_{1}^{(i)}, Y_{2}^{(i)}, \ldots, Y_{n}^{(i)}\right),
\end{gathered}
$$

could be found, with limit points

$\left(\xi_{1}, \xi_{2}, \ldots, \xi_{m}: \eta_{1}, \eta_{2}, \ldots, \eta_{n}\right), \quad\left(\xi_{1}, \xi_{2}, \ldots, \xi_{m}: H_{1}, H_{2}, \ldots, H_{n}\right)$

which lie in the region $\mathfrak{C}$. Since by $C$ ) no two points in $\mathfrak{C}$ have the same projection in $\mathfrak{G}$ it follows that $\left(\eta_{1}, \eta_{2}, \ldots, \eta_{n}\right)$ and $\left(H_{1}, H_{2}, \ldots, H_{n}\right)$ are the same. At the point $\left(\xi_{1}, \xi_{2}, \ldots, \xi_{m}: \eta_{1}, \eta_{2}, \ldots, \eta_{n}\right)$ the usual theorems on implicit functions $\dagger$ can be applied to equations (1) on account of $A), B), D$ ), and it follows that to any point in the neighborhood of $\left(\xi_{1}, \xi_{2}, \ldots, \xi_{m}\right)$ there can correspond but one set of solutions in the neighborhood of $\left(\xi_{1}, \xi_{2}, \ldots, \xi_{m}: \eta_{1}, \eta_{2}, \ldots, \eta_{n}\right)$. Thus the existence of the sequences (3) is contradicted.

The constant $\epsilon$ can be still further restricted if necessary so that the functional determinant $\partial\left(f_{1}, f_{2}, \ldots, f_{n}\right) / \partial\left(y_{1}, y_{2}, \ldots, y_{n}\right)$ is different from zero in $\mathfrak{E}_{e}$, on account of $D$ ).

The next step is to select a constant $\delta \leqq \epsilon$ so that to each point in $\mathfrak{G}_{\delta}$ there corresponds at least one solution in the region $\mathfrak{E}_{\mathrm{e}}$. Suppose that $P$ is any point of the set $\mathfrak{E}$, and $Q$ its projection in $\mathfrak{S}$. Then by the theorems on implicit functions referred to above, a constant $\delta$ can be found such that to each point $\left(x_{1}, x_{2}, \ldots, x_{m}\right)$ in the neighborhood $Q_{\delta}$ there corresponds a solution of equations (1) lying in $P_{e}$ and therefore in $\mathfrak{E}_{e} \cdot A$ constant $\delta$ can be selected which will be effective in this way uniformly for all the points $P$ of $\mathfrak{E}$. Otherwise a sequence $\left\{P_{n}\right\}$ would exist in $\mathfrak{E}$, with limit point $\pi$, for which the corresponding constants $\delta_{n}$ would have the limit zero. This is, however, impossible, since there is a value $\delta_{\pi}>0$ for the limit point $\pi$.

By combining these results it follows that to any point in the region $\mathfrak{W}_{\delta}$ there corresponds one and but one solution of equations (1) in the neighborhood $\mathbb{E}_{e}$. Since the functional determinant does not vanish in $\mathfrak{E}_{e}$ it follows from the usual theorems concerning implicit functions that the functions (2) so defined over the region $\mathfrak{G}_{\delta}$ will be surely of class $C$ under the conditions on the functions $f_{i}$ stated under $A$ ), and of class $C^{(n)}$ when the functions $f_{\mathfrak{i}}$ are of class $C^{(n)}$ in $\mathfrak{A}$.

* For the details of the construction of these sequences see BolzA, loc. cit., p. 161.

† See, for example, Osgood, Lehrbuch der Funktionentheorie, vol. 1, p. 52; or GoursatHeDrick, A Course in Analysis, vol. 1, p. 45 and footnote. 


\section{§2. The field about a point.}

The equations of the extremals through a given point $P\left(x_{0}, y_{0}, z_{0}\right)$ may be taken in the form*

$$
x=\phi(s, \xi, \eta, \zeta), \quad y=\psi(s, \xi, \eta, \zeta), \quad z=\chi(s, \xi, \eta, \zeta),
$$

where the functions $\phi, \psi, \chi$ and their first partial derivatives with respect to $s$ are of class $C^{\prime}$ in the neighborhood of the values of $s, \xi, \eta, \zeta$ defined by the conditions

$$
s=0, \quad \xi^{2}+\eta^{2}+\zeta^{2}=1 .
$$

For $s=0$ the identities

$$
\begin{aligned}
& \phi(0, \xi, \eta, \zeta)=x_{0}, \quad \psi(0, \xi, \eta, \zeta)=y_{0}, \quad \chi(0, \xi, \eta, \zeta)=z_{0}, \\
& \phi_{s}(0, \xi, \eta, \zeta)=\xi, \quad \psi_{\imath}(0, \xi, \eta, \zeta)=\eta, \quad \chi_{s}(0, \xi, \eta, \zeta)=\zeta
\end{aligned}
$$

hold, and the identity

$$
\phi \phi_{s}+\psi \psi_{s}+\chi \chi_{\mathrm{s}}=0
$$

is satisfied for all arguments. If

$$
\xi^{2}+\eta^{2}+\zeta^{2}=1,
$$

it follows that $s$ is the length of are measured along the extremal from the point $P$, so that

$$
\phi_{s}^{2}+\psi_{s}^{2}+\chi_{s}^{2}=1,
$$

and $\xi, \eta, \zeta$ are the direction cosines of the tangent to the extremal at $P$.

It is desired to show that two positive constants $d$, e exist such that to any point $(x, y, z)$ in the region. $P_{d}^{\prime}, \dagger$

$$
0<\left|x-x_{0}\right|<d, 0<\left|y-y_{0}\right|<d, 0<\left|z-z_{0}\right|<d,
$$

there exists one and but one solution $(x, y, z: s, \xi, \eta, \zeta)$ of the equations

$$
\xi^{2}+\eta^{2}+\zeta^{2}=1, \phi(s, \xi, \eta, \zeta)=x, \psi(s, \xi, \eta, \zeta)=y, \chi(s, \xi, \eta, \zeta)=z
$$

for which $(s, \xi, \eta, \zeta)$ is in the region $\mathfrak{S}_{e}^{\prime}$ defined by the conditions

$$
0<s<e, \quad \xi^{2}+\eta^{2}+\zeta^{2}=1 .
$$

In terms of the polar coördinates $R, A, B, C$ defined by the equations

$$
x-\ddot{x}_{0}=R A, y-y_{0}=R B, z-z_{0}=R C, R>0, A^{2}+B^{2}+C^{2}=1,
$$

* See Mason and Bliss, loc. cit., p. 444.

$\dagger$ Note that $P_{d}^{\prime}$ is the region $P_{d}$ as defined in a preceding footnote, with the point $\left(x_{0}, y_{0}, z_{0}\right)$ ercluded. 
the equations (7) become

(8) $\xi^{2}+\eta^{2}+\zeta^{2}=1, \phi(s, \xi, \eta, \zeta)=R A, \psi(s, \xi, \eta, \zeta)=R B, \chi(s, \xi, \eta, \zeta)=R C$.

If $s$ is replaced by $\sigma$, where $s=R \sigma$, they take the form

$$
\begin{aligned}
\xi^{2}+\eta^{2}+\zeta^{2} & =1, \\
\frac{1}{R}\left[\phi(R \sigma, \xi, \eta, \zeta)-x_{0}\right] & =A, \\
\frac{1}{R}\left[\psi(R \sigma, \xi, \eta, \zeta)-y_{0}\right] & =B, \\
\frac{1}{R}\left[\chi(R \sigma, \xi, \eta, \zeta)-z_{0}\right] & =C .
\end{aligned}
$$

By Taylor's formula with the integral form of the remainder term * and the identities (4), it follows that

$$
\phi(s, \xi, \eta, \zeta)-x_{0}=s \int_{0}^{1} \phi_{s}(t s, \xi, \eta, \zeta) d t,
$$

with similar equations for $\psi$ and $\chi$, so that when the first members of equations (9) are defined for $R=0$ by their limiting values, these equations reduce, for $R=0$, to

$$
\xi^{2}+\eta^{2}+\zeta^{2}=1, \sigma \xi=A, \sigma \eta=B, \sigma \zeta=C .
$$

It is evident then that equations (9) have the solutions

$$
(R, A, B, C: \sigma, \xi, \eta, \zeta)=(0, A, B, C: 1, \xi, \eta, \zeta), A^{2}+B^{2}+C^{2}=1,
$$

and the projection of $\mathfrak{C}$ in terms of the polar coördinates is the region defined by the conditions

$$
R=0, \quad A^{2}+B^{2}+C^{2}=1 .
$$

From equation (10) and its analogues for $\psi$ and $\chi$, it follows that the left members of equations (9) are of class $C^{\prime}$ in the neighborhood of $\mathfrak{E}$, and in $\mathfrak{E}$ their functional determinant with respect to $\sigma, \xi, \eta, \zeta$ is calculated from (11) to be $2 \sigma^{2}=2$. Hence Bolza's theorem is applicable, and it follows that a constant $\epsilon$ exists such that no two solutions of equations $(9)$ in the region $\mathfrak{E}_{e}$ have the same projection in $\mathfrak{H}_{\epsilon}$, and a second constant $\delta \leqq \epsilon$, can be chosen so that to any $(R, A, B, C)$ in $\mathfrak{W}_{\delta}$ there corresponds a set of values

$$
\sigma(R, A, B, C), \xi(R, A, B, C), \eta(R, A, B, C), \zeta(R, A, B, C)
$$

which with $(R, A, B, C)$ satisfy equations $(9)$ and lie in $\mathbb{C}_{e} \cdot$ The functions (12) are of class $C^{\prime}$ and reduce to $1, A, B, C$ for $R=0$.

* See C. JoRdan, Cours d'Analyse, rol. 1, p. 245.

Trans. Am. Math. Soc. 28 
With these results in mind it is possible to show the existence of a constant $e$ such that no two sets of values $(s, \xi, \eta, \zeta)$ in $\mathcal{S}_{c}^{\prime}$ can define by means of equations $(7)$ the same point $(x, y, z)$, or what is equivalent, the same values $(R, A, B, C)$. In fact, for any solution of equations (8) the value of $R$ is given in terms of $(s, \xi, \eta, \zeta)$ by means of the formula

$$
R^{2}=\left[\phi-x_{0}\right]^{2}+\left[\psi-y_{0}\right]^{2}+\left[x-z_{0}\right]^{2},
$$

and from equations $(10)$ and (4) it is evident therefore that the quotient $s / R$ is a continuous function of $s, \xi, \eta, \zeta$ which reduces to 1 for $s=0$. Similarly $A, B, C$ in equations (8) are seen to be continuous functions of $s, \xi, \eta, \zeta$ which take the values $\xi, \eta, \zeta$, respectively. for $s=0$. Hence if $e$ is sufficiently small, no two sets of values $(s, \xi, \eta, \zeta)$ in $\mathcal{S}_{e}^{\prime}$ can define the same $(x, y, z)$. Otherwise equations (9) would have two solutions $(R, A, B, C: \sigma, \xi, \eta, \zeta)$ in $\mathbb{E}$ corresponding to the same $(R, A, B, C)$.

Let $\delta$ be taken so small that the values

$$
s=R \sigma(R, A, B, C), \xi(R, A, B, C), \eta(R, A, B, C), \zeta(R, A, B, C)
$$

corresponding to any $(R, A, B, C)$ in $\mathfrak{W}_{\delta}$, lie in $\mathfrak{S}_{\epsilon}$. If $d=\delta / \sqrt{ } 3$, then to any point $(x, y, z)$ in $P_{d}^{\prime}$ there will correspond values of $(R, A, B, C)$ in $\mathfrak{G}_{\delta}$, and consequently a solution of equations (7) for which $(s, \xi, \eta, \zeta)$ lies in $\mathfrak{S}_{e}^{\prime}$. By the preceding paragraph this solution will be unique in $\mathfrak{S}_{e}^{\prime}$.

The functions

$$
s(x, y, z), \xi(x, y, z), \eta(x, y, z), \zeta(x, y, z)
$$

so defined have the following properties :

1) In the neighborhood $P_{1}^{\prime}$ all are of class $C^{\prime}$.

2) In $P_{d}$ the function $s(x, y, z)$ is continuous and has the value 0 as $\left(x_{0}, y_{0}, z_{0}\right)$.

3) Let $C$ be an arc of class $C^{\prime}$,

$$
x=f(t), \quad y=g(t), \quad z=h(t), \quad \cdot(0 \leqq t \leqq T),
$$

which passes through $P\left(x_{v}, y_{0}, z_{0}\right)$ for $t=0$. The function $s(t)$ defined by substituting the values $(C)$ in the expressions (14) has at $t=0$ the derivative

$$
\boldsymbol{s}^{\prime}(0)=\sqrt{f^{\prime 2}(0)+g^{\prime 2}(0)+{h^{\prime 2}}^{2}(0)},
$$

and the corresponding functions $\xi(t), \eta(t), \zeta(t)$ are continuous and reduce for $t=0$ to the direction cosines $A_{0}, B_{, 0}, C_{0}$, of the positive tangent to $C$ at $P$, respectively.

The first two properties follow immediately from the nature of the functions 
(2) and of the polar coördinates $R, A, B, C$ as functions of $x, y, z$. For points on the curve $C$

$$
\begin{aligned}
R^{2} & =\left[f-x_{0}\right]^{2}+\left[g-y_{0}\right]^{2}+\left[h-z_{0}\right]^{2}, \\
A & =\frac{f-x_{0}}{R}, \quad B=\frac{g-y_{0}}{R}, \quad C=\frac{h-z_{0}}{R} .
\end{aligned}
$$

Hence by applying Taylor's formula to the differences $f-x_{0}, g-y_{0}, h-z_{0}$ it follows that

$$
\lim _{t=0} \frac{R}{t}=\sqrt{{f^{\prime 2}(0)+g^{\prime 2}(0)+{h^{\prime}}^{2}(0)}^{2}}
$$

and also that $A, B, C$ approach the limits $A_{0}, B_{0}, C_{0}$. From the equations (13) and the properties of the functions (12) it is then easy to prove the statement 3).

The proof which has been made applies to the construction of a field in the neighborhood of a single point $\left(x_{0}, y_{0}, z_{0}\right)$. It is important, for the proof of Hilbert's theorem concerning the existence of an extremal joining two given points in space, to know that such fields can be constructed uniformly about the points of a finite closed region $\Re$. The equations of the extremals through points of $\Re$ have the form *

$$
\begin{aligned}
& x=\phi\left(s, x_{0}, y_{0}, z_{0}, \xi, \eta, \zeta\right), \\
& y=\psi\left(s, x_{0}, y_{0}, z_{0}, \xi, \eta, \zeta\right), \\
& z=\chi\left(s, x_{0}, y_{0}, z_{0}, \xi, \eta, \zeta\right),
\end{aligned}
$$

where $\phi, \psi, \chi$ and their derivatives for $s$ are of class $C^{\prime}$ in the neighborhood of the domain defined by the conditions

$$
\left(x_{0}, y_{0}, z_{0}\right) \text { in } R, s=0, \xi^{2}+\eta^{2}+\zeta^{2}=1 .
$$

For $s=0$ the identities (4) hold with respect to all seven arguments, and the identity (6) is a consequence of equation (5).

The equations corresponding to (9) can be set up in the same manner as before, but they contain now the additional arguments $x_{0}, y_{0}, z_{0}$. The region corresponding to $\mathfrak{C}$ for these equations is the region

(永) $\left(x_{0}, y_{0}, z_{0}: 0, A, B, C: 1, A, B, C\right),\left(x_{0}, y_{0}, z_{0}\right)$ in $R, A^{2}+B^{2}+C^{2}=1$, and the region corresponding to $\mathfrak{S}$ is

$(\overline{\mathfrak{G}}) \quad\left(x_{0}, y_{0}, z_{0}: 0, A, B, C\right),\left(x_{0}, y_{0}, z_{0}\right)$ in $R, A^{2}+B^{2}+C^{2}=1$.

\footnotetext{
* Mason AND BLISs, loc. oit., p. 444.
} 
The constants $\delta$ and $\epsilon$ can be determined so that no two solutions of equations (9) in $\overline{\mathfrak{E}}_{\mathrm{e}}$, have the same projection in $\overline{\mathfrak{W}}_{\mathrm{e}}$, and so that to each point in $\overline{\mathfrak{W}}_{\delta}$ there corresponds at least one solution in $\overline{\mathfrak{E}}_{\mathrm{e}}$, the variables $\sigma, \xi, \eta, \zeta$ being now functions of class $C^{\prime}$ of $x_{0}, y_{0}, z_{0}, R, A, B, C$ in $\overline{\mathfrak{G}}_{\delta}$ which reduce to $(1, A$, $B, C)$ for points in $\overline{\mathfrak{H}}$.

Each solution $\left(x_{0}, y_{0}, z_{0}: R, A, B, C: s, \xi, \eta, \zeta\right)$ of equations (8) defines as before a solution $\left(x_{0}, y_{0}, z_{0}: R, A, B, C: \sigma, \xi, \eta, \zeta\right)$ of equations (9) whose elements are continuous functions of $x_{0}, y_{0}, z_{0}, s, \xi, \eta, \zeta$ reducing to $\left(x_{0}, y_{0}, z_{0}: 0, A, B, C: 1, \xi, \eta, \zeta\right)$ when $\left(x_{0}, y_{0}, z_{0}\right)$ is in $R$ and $(s, \xi, \eta, \zeta)$ is in the region $S$. Hence the constant $e$ can be chosen so that the solution of equation of equation (9) always lies in $\overline{\mathfrak{E}}_{\mathrm{e}}$ when $\left(x_{0}, y_{0}, z_{0}\right)$ is in $\mathfrak{R}$ and $(s, \xi, \eta, \zeta)$ in $\mathcal{S}_{e}^{\prime}$, and consequently for any fixed but arbitrarily chosen point $\left(x_{0}, y_{0}, z_{0}\right)$ in $\Re$ no two sets of values $(s, \xi, \eta, \zeta)$ in $\mathcal{S}_{e}^{\prime}$ could define the same $(R, A, B, C)$. Otherwise equations (9) would have two solutions in $\overline{\mathbb{E}}_{\mathrm{e}}$ corresponding to the same point in $\overline{\mathfrak{H}}_{\epsilon}$.

The constant $d=\delta / \sqrt{ } 3$ can similarly be chosen so that for any point $P$ $\left(x_{0}, y_{0}, z_{0}\right)$ in $\Re$ the values $(R, A, B, C)$ in the region $P_{a}^{\prime}$ about $P$ define solutions $(x, y, z: s, \xi, \eta, \zeta)$ of equations (15) and (5) for which $(s, \xi, \eta, \zeta)$ is unique in $\Im_{e}^{\prime}$.

Hence constants $d, e$ can be chosen uniformly for the region $\Re$ so that to any point $(x, y, z)$ in the neighborhood $P_{d}^{\prime}$,

$$
0<\left|x-x_{0}\right|<d, \quad 0<\left|y-y_{0}\right|<d, \quad 0<\left|z-z_{0}\right|<d,
$$

of a point $P\left(x_{0}, y_{0}, z_{0}\right)$ of $\mathfrak{R}$ there exists one and but one solution of equations (15), for which $(s, \xi, \eta, \zeta)$ is in the region

$$
0<s<e, \quad \xi^{2}+\eta^{2}+\zeta^{2}=1 .
$$

The functions of $x_{0}, y_{0}, z_{0}, x, y, z$ so determined are of class $C^{\prime}$ for $\left(x_{0}, y_{0}, z_{0}\right)$ in $\Re$ and $(x, y, z)$ in $P_{d}^{\prime}$, and have the properties described above when $\left(x_{0}, y_{0}, z_{0}\right)$ is fixed.

From this result is derived the following geometrical theorem:

$A$ constant $\delta>0$ can be determined so that through any point $Q \neq P$ in $a$ sphere of radius $\delta$ about $P$, where $P$ is an arbitrarily selected point of $R$, there passes one and but one extremal arc (15) which joins $P$ with $Q$ and lies entirely within the sphere.

Along an extremal, $R$ is a continuous function of $x_{0}, y_{0}, z_{0}, s, \xi, \eta, \zeta$ and has a derivative $d R / d s$ which reduces to 1 for $s=0$. To make sure that the extremal arc joining $P$ with $Q$ lies entirely within the sphere it is therefore only necessary to choose $e$ so small that $d R / d s$ remains different from zero for $\left(x_{0}, y_{0}, z_{0}\right)$ in $R$ and $(s, \xi, \eta, \zeta)$ in the region $S_{e}^{\prime}$. Then the corresponding constant $\delta$ will be the one desired. 
§3. The construction of a field of: extremals to which a given arc $\mathfrak{L}$ is transversal.

Consider an arc $\mathfrak{L}$

$$
x=X(u), y=Y(u), z=Z(u), u_{1} \leqq u \leqq u_{2},
$$

which has the properties that it is part of a curve of class $C^{\prime \prime}$, that it does not intersect itself, and that the parameter $u$ is the length of arc. Let

$$
\alpha, \beta, \gamma: l, m, n: \lambda, \mu, \nu
$$

be the direction cosines of the tangent, principal normal, and binormal, respectively.

In each half-plane through a tangent to $\mathfrak{L}$ and containing the direction

$$
l \cos v+\lambda \sin v, m \cos v+\mu \sin v, n \cos v+\nu \sin v
$$

normal to the curve, there is one and but one direction $\xi, \eta, \zeta$ distinct from the

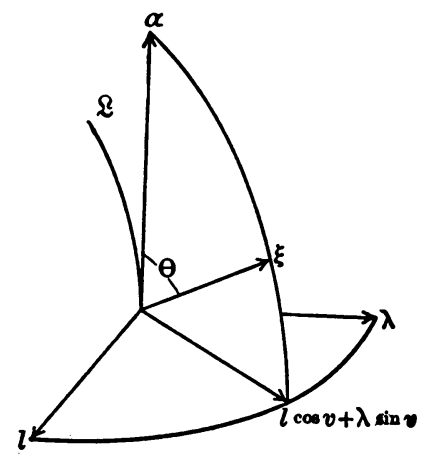

Fig. 1.

tangent, to which the curve $\mathfrak{R}$ is transversal.* The angle $\theta(0<\theta<\pi)$ (see Fig. 1) determining this direction, is a function of $u, v$ which is periodic in $v$ with period $2 \pi$, and the values of $\xi, \eta, \zeta$ are

$$
\begin{aligned}
& \xi=\alpha \cos \theta+(l \cos v+\lambda \sin v) \sin \theta, \\
& \eta=\beta \cos \theta+(m \cos v+\mu \sin v) \sin \theta, \\
& \zeta=\gamma \cos \theta+(n \cos v+\nu \sin v) \sin \theta .
\end{aligned}
$$

The extremals to which $\mathfrak{L}$ is transversal are found by substituting the functions $X(u), Y(u), Z(u)$ defining $\mathbb{R}$, in place of $x_{0}, y_{0}, z_{0}$ in equations (15) of

* Bliss and Mason, loc. cit., p. 463. 
$\S 2$, and the values $(2)$ in place of $\xi, \eta, \zeta$. Then the equations of the extremals have the form

$$
x=\phi(s, u, v), \quad y=\psi(s, u, v), \quad z=\chi(s, u, v),
$$

where the functions $\phi, \psi, \chi$ can be supposed of class $C^{\prime \prime}$ in a certain neighborhood of the region $\subseteq$ defined by the conditions

$$
s=0, \quad u_{1} \leqq u \leqq u_{2}, \quad v \text { any value },
$$

and are periodic in $v$ with period $2 \pi$. The parameter $s$ is the length of arc, so that the identity

$$
\phi_{s}^{2}+\psi_{s}^{2}+\chi_{s}^{2} \equiv 1
$$

is satisfied for all arguments. For $s=0$,

(3) $\phi(0, u, v) \equiv X(u), \quad \psi(0, u, v) \equiv Y(u), \quad \chi(0, u, v) \equiv Z(u)$,

$$
\phi_{s}(0, u, v) \equiv \alpha \cos \theta+(l \cos v+\lambda \sin v) \sin \theta,
$$

$$
\begin{aligned}
& \psi_{\mathfrak{s}}(0, u, v) \equiv \beta \cos \theta+(m \cos v+\mu \sin v) \sin \theta, \\
& \chi_{s}(0, u, v) \equiv \gamma \cos \theta+(n \cos v+\nu \sin v) \sin \theta,
\end{aligned}
$$

and since $\theta$ is between 0 and $\pi$, it follows from the relations which are satisfied by the mutually orthogonal directions (1) that

$$
\begin{aligned}
\cos \theta=\alpha \phi_{s}(0, u, v)+\beta \psi_{s}(0, u, v)+ & \gamma \chi_{s}(0, u, v), \\
\sin \theta=(l \cos u+\lambda \sin v) \phi_{s}(0, u, v)+ & (m \cos v+\mu \sin v) \psi_{s}(0, u, v) \\
& +(n \cos v+\nu \sin v) \chi_{s}(0, u, v)>0 .
\end{aligned}
$$

If $A, B, C$ are the cosines of a direction not coincident with the tangent to $\mathcal{L}$, then there is one and but one value of $v$ (modulo $2 \pi$ ) for which the tangent to the extremal $E$ is coplanar with $A, B, C$ and the tangent to $\mathfrak{R}$, and which lies on the same side of the tangent to $\mathfrak{R}$ with $A, B, C$. For in order that these conditions may be satisfied the determinant

$$
\left|\begin{array}{lll}
\phi_{s}(0, u, v) & \alpha & A \\
\psi_{\triangleleft}(0, u, v) & \beta & B \\
\chi_{s}(0, u, v) & \gamma & C
\end{array}\right|
$$

must vanish, and the expression

$$
\cos \omega=A(l \cos v+\lambda \sin v)+B(m \cos v+\mu \sin v)+C(n \cos v+\nu \sin v)
$$

must be positive. With the help of equations (4) the former condition is seen to determine the ratio of $\cos v$ and $\sin v$, and the latter determines their signs, 
uniquely. It follows that if $v$ and $v^{\prime}$ are not congruent modulo $\pi$ the determinant

$$
\left|\begin{array}{lll}
\phi_{s}(0, u, v) & \alpha & \phi_{s}\left(0, u, v^{\prime}\right) \\
\psi_{s}(0, u, v) & \beta & \psi_{c}\left(0, u, v^{\prime}\right) \\
\chi_{s}(0, u, v) & \gamma & \chi_{o}\left(0, u, v^{\prime}\right)
\end{array}\right|=\sin \left(v-v^{\prime}\right)
$$

is always different from zero.

By substitution of the values for $\phi_{c}$ and $\phi_{s 0}$ from equations (4) a simple computation shows that when $v \equiv v^{\prime}(\operatorname{modulo} \pi)$

$$
\left|\begin{array}{lll}
\phi_{s}(0, u, v) & \alpha & \phi_{s v}\left(0, u, v^{\prime}\right) \\
\psi_{s}(0, u, v) & \beta & \psi_{s v}\left(0, u, v^{\prime}\right) \\
\chi_{s}(0, u, v) & \gamma & \chi_{s v}\left(0, u, v^{\prime}\right)
\end{array}\right|= \pm \sin \theta \sin \theta^{\prime} \neq 0 .
$$

The notation $\mathfrak{R}_{\delta}$ will be used to denote the $\delta$-neighborhood of the curve $\mathfrak{R}$, and $\Omega_{\delta}^{\prime}$ will denote the points of this set exclusive of those on the curve of which $\mathfrak{R}$ is a part. In the following pages any two points $(s, u, v)$ and $\left(s^{\prime}, u^{\prime}, v^{\prime}\right)$ are regarded as identical when $s=s^{\prime}, u=u^{\prime}$, and $v$ is congruent to $v^{\prime}$ modulo $2 \pi$ $\left(v \equiv v^{\prime}\right)$, otherwise distinct. The region $S_{\text {, is }}$

$$
0 \leqq s \leqq \epsilon, \quad u_{1}-\epsilon \leqq u \leqq u_{2}+\epsilon, \quad v \text { any value, }
$$

and $\Im_{e}^{\prime}$ is $\varsigma_{e}$ exclusive of the points for which $s=0$.

The purpose of the present section is the proof of the following statement:

$T w o$ positive constants $\delta, \epsilon$ can be determined 80 that to any point $(x, y, z)$ in the neighborhood $\mathfrak{L}_{0}^{\prime}$ there corresponds one and but one solution $(x, y, z: s, u, v)$ of the equations $(E)$ for which the values $(s, u, v)$ lie in the region $S_{.}^{\prime}$.

In the first place $\epsilon$ can be chosen so small that no two points $(s, u, v)$, $\left(s^{\prime}, u^{\prime}, v^{\prime}\right)$ in the region $\mathcal{S}_{e}$ define the same point $(x, y, z)$ by means of the equations $(E)$, unless $s=s^{\prime}=0, u=u^{\prime}$. In other words, no two of the extremals $(E)$ can intersect for values of $(s, u, v)$ in $S_{\text {, unless they pass }}$ through the same point $P$ of the curve of which $\mathfrak{Q}$ is a part, in which case $P$ is the only point of intersection, and furthermore no extremal intersects itself. For if these statements were not true, a sequence

$$
\begin{gathered}
\left\{s_{i}, u_{i}, v_{i}: s_{i}^{\prime}, u_{i}^{\prime}, v_{i}^{\prime}\right\} \\
\left(s_{i}, s_{i}^{\prime}\right) \neq(0,0), \quad\left(s_{i}, u_{i}, v_{i}\right) \neq\left(s_{i}^{\prime}, u_{i}^{\prime}, v_{i}^{\prime}\right)
\end{gathered}
$$

of solutions of the equations

$$
\begin{aligned}
& \phi(s, u, v)=\phi\left(s^{\prime}, u^{\prime}, v^{\prime}\right), \\
& \psi(s, u, v)=\phi\left(s^{\prime}, u^{\prime}, v^{\prime}\right), \\
& \chi(s, u, v)=\chi\left(s^{\prime}, u^{\prime}, v^{\prime}\right),
\end{aligned}
$$


could be determined with limiting values of the form

$$
\left(0, u_{0}, v_{0}: 0, u_{0}^{\prime}, v_{0}^{\prime}\right), \quad u_{1} \leqq u_{0} \leqq u_{2}, \quad u_{1} \leqq u_{0}^{\prime} \leqq u_{2},
$$

which would also satisfy the equations. Since $\mathfrak{L}$ does not intersect itself, it follows from the identities (3) that $u_{0}$ and $u_{0}^{\prime}$ must be the same.

With the help of the identities (3) any one of the equations (8), say the first, can be written in the form

$$
\left[\phi(s, u, v)-\phi\left(0, u^{\prime}, v\right)\right]-\left[\phi\left(s^{\prime}, u^{\prime}, v^{\prime}\right)-\phi\left(0, u^{\prime}, v^{\prime}\right)\right]=0,
$$

or by Taylor's formula

$$
\begin{array}{r}
s \int_{0}^{1} \phi_{c}\left[t s, u^{\prime}+t\left(u-u^{\prime}\right), v\right] d t+\left(u-u^{\prime}\right) \int_{0}^{1} \phi_{u}\left[t s, u^{\prime}+t\left(u-u^{\prime}\right), v\right] d t \\
-s^{\prime} \int_{0}^{1} \phi_{d}\left(t s^{\prime}, u^{\prime}, v^{\prime}\right) d t=0 .
\end{array}
$$

The three equations so found would have solutions $s_{i}, u_{i}-u_{i}^{\prime}, s_{i}^{\prime}$ not all zero provided by the sequence (7). Their determinant with respect to the arguments $s, u-u^{\prime}, s^{\prime}$ outside the integral signs, is a continuous function of $s, u, v, s^{\prime}, u^{\prime}, v^{\prime}$ which must vanish for the arguments (7), and therefore also for the arguments (9). But for the latter it takes the form (5), showing that $v_{0}$ must be congruent to $v_{0}^{\prime}$ modulo $\pi$, since the determinant (5) vanishes for such values only.

From the identities (3) it appears that the function $\phi_{v}(0, u, v)$ is identically zero, and therefore

$$
\phi_{v}(s, u, v)=s \int_{0}^{1} \phi_{v v}(\tau s, u, v) d \tau .
$$

By an application of Taylor's formula, and then of equation (10), the first of equations (8) can be written again in the form

$$
\begin{aligned}
\phi(s, u, v)-\phi\left(s^{\prime}, u^{\prime}, v^{\prime}\right)= & \phi(s, u, v)-\phi\left(s^{\prime}, u^{\prime}, v\right)+\phi\left(s^{\prime}, u^{\prime}, v\right)-\phi\left(s^{\prime}, u^{\prime}, v^{\prime}\right) \\
= & \left(s-s^{\prime}\right) \int_{0}^{1} \phi_{v}\left[s^{\prime}+t\left(s-s^{\prime}\right), u^{\prime}+t\left(u-u^{\prime}\right), v\right] d t \\
& \left.+\left(u-u^{\prime}\right) \int_{0}^{1} \phi_{u}\left[s^{\prime}+t\left(s-s^{\prime}\right), u^{\prime}+t\left(u-u^{\prime}\right), v\right)\right] d t \\
& +\left(v-v^{\prime}\right) s^{\prime} \int_{0}^{1} \int_{0}^{1} \phi_{v o}\left[\tau s^{\prime}, u^{\prime}, v^{\prime}+t\left(v-v^{\prime}\right)\right] d \tau d t
\end{aligned}
$$

The three equations so found have a determinant with respect to the quantities $s-s^{\prime}, u-u^{\prime},\left(v-v^{\prime}\right) s^{\prime}$ outside the integral signs, which is a continuous function reducing to (6) for the arguments $\left(0, u, v: 0, u, v^{\prime}\right)$. At the limiting values (9) this determinant is different from zero since $u_{0}=u_{0}^{\prime}, v_{0} \equiv v_{\text {? }}^{\prime}$ (modulo $\pi$ ). 
Hence for a sufficiently large value of $i$ any quantities $s_{i}-s_{i}^{\prime}, u_{i}-u_{i}^{\prime}$, $\left(v_{i}-v_{i}^{\prime}\right) s_{i}^{\prime}$ which satisfy these equations must all vanish, and by interchanging $(s, u, v)$ with $\left(s^{\prime}, u^{\prime}, v^{\prime}\right)$ in equation (8) it follows that $\left(v_{i}^{\prime}-v_{i}\right) s_{i}$ must also be zero. If the sequence ( 7$)$ existed, therefore, the points $\left(s_{i}, u_{i}, v_{i}\right)$ and $\left(s_{i}^{\prime}, u_{i}^{\prime}, v_{i}^{\prime}\right)$ would have to coincide, or else $s_{i}=s_{i}^{\prime}, u_{i}=u_{i}^{\prime}$, and $s_{i}$ and $s_{i}^{\prime}$ would have to vanish simultaneously, for each sufficiently large value of $i$. But these are the properties which the sequence (7) was assumed not to have, and consequently its existence is impossible.

The constant $\epsilon$ can be subjected to a second restriction, if necessary, to the effect that for all values of $(s, u, v)$ in $\varsigma_{e}^{\prime}$ the functional determinant of equations $(E)$ with respect to $s, u, v$ is different from zero. With the help of equation (10) this determinant takes the form

$$
\left|\begin{array}{lll}
\phi_{0} & \phi_{u} & \phi_{v} \\
\psi_{v} & \psi_{u} & \psi_{v} \\
\chi_{v} & \chi_{u} & \chi_{v}
\end{array}\right|=s V(s, u, v),
$$

where $V$ is a continuous function in the region $\varsigma_{e}$, different from zero for $s=0$ on account of the condition (6). Hence if $\epsilon$ is properly chosen $V$ will remain different from zero in $\varsigma_{e}^{\prime}$.

Suppose now that $\epsilon$ has been chosen so as to satisfy the two restrictions described above. The points $(x, y, z)$ of the extremals $(E)$ for which $u=u_{1}-\epsilon$ or $u=u_{2}+\epsilon$, or for which $s=\epsilon$, form a closed set which has no point in common with the arc $\mathfrak{R}$. Let $\delta \sqrt{3}$ be the minimum distance between the points of this set and the points of $\mathfrak{R}$. Then any solution $(x, y, z: s, u, v)$ of equations $(E)$, corresponding to a point $P(x, y, z)$ in $\mathfrak{R}_{\delta}^{\prime}$, has values $(s, u, v)$ in the interior of $\mathfrak{S}_{e}^{\prime}$. For $P$ is not on $\mathfrak{L}$ and therefore can not correspond to a set $(s, u, v)$ for which $s=0$, and its distance from $\mathfrak{R}$ is less than $\delta \sqrt{3}$, so that, on account of the way in which $\delta$ has just been selected, no one of the three conditions $u=u_{1}-\epsilon, u=u_{3}+\epsilon, s=\epsilon$ can be satisfied.

From the first property of the constant $\epsilon$ it follows, furthermore, that $P$ can not correspond to more than one set $(s, u, v)$ in $\varsigma^{\prime}$.

On the other hand, each point $P$ in $\mathfrak{R}_{\delta}^{\prime}$ corresponds to at least one set of values $(s, u, v)$ in the interior of $\varsigma_{e}$. For consider any particular point $P^{\prime}\left(x^{\prime}, y^{\prime}, z^{\prime}\right)$ in $\Omega_{\delta}^{\prime}$ which has corresponding to it the values $\left(s^{\prime}, u^{\prime}, v^{\prime}\right)$. Join $P^{\prime}$ with $P$ by an $\operatorname{arc} D$

$$
x=x(t), \quad y=y(t), \quad z=z(t), \quad t^{\prime} \leqq t \leqq T
$$

of class $C^{\prime}$ which lies entirely within $\mathfrak{R}_{\delta}^{\prime}$. The functional determinant with respect to $s, u, v$ of the first members of the equations

$$
\phi(s, u, v)=x(t)
$$$$
\psi(s, u, v)=y(t)
$$$$
\chi(s, u, v)=z(t)
$$ 
is different from zero at $\left(s^{\prime}, u^{\prime}, v^{\prime}\right)$ on account of the second property of the constant $\epsilon$, and these equations have the particular solutions $\left(x^{\prime}, y^{\prime}, z^{\prime}, t^{\prime}\right)$. It follows that equations (11) have continuous solutions $s(t), u(t), v(t)$ in the neighborhood of $t^{\prime}$, which can be continued along the arc $D$ up to the point $P$, so that $P$ also corresponds to a solution $(x, y, z: s, u, v)$ of equations $(E)$ for which $(s, u, v)$ necessarily lies in $\mathcal{S}_{c}^{\prime}$. Otherwise there would be an upper bound $\tau$ to the values of $t$ which could be reached by continuation, with a corresponding solution $\left(s_{\tau}, u_{\tau}, v_{\tau}, \tau\right)$ of equations (11) for which $\left(s_{\tau}, u_{\tau}, v_{\tau}\right)$ would lie in $\varsigma_{e}^{\prime}$. The functional determinant of equations (11) would still be different from zero at $\left(s_{\tau}, u_{\tau}, v_{\tau}\right)$ and consequently $\tau$ could not be the upper bound described.

It has therefore been shown that to each point $(x, y, z)$ in $\mathfrak{R}_{\delta}^{\prime}$ there corresponds a solution $(x, y, z: s, u, v)$ of equations $(E)$ for which $(s, u, v)$ lies in $S_{\epsilon}^{\prime}$, and on account of the way in which $\epsilon$ was chosen this solution is unique. The functions

$$
s(x, y, z), u(x, y, z), v(x, y, z)
$$

which with $x, y, z$ solve equations $(E)$ have the following properties in the region $\mathfrak{R}_{\delta}^{\prime}$ :

1) They are all three of class $C^{\prime}$ in $\mathfrak{R}_{\delta}^{\prime}$.

2) In $\mathfrak{L}_{\delta}$ the first two are continuous and for a point $\left(x_{0}, y_{0}, z_{0}\right)$ on $\mathfrak{L}$ corresponding to the parameter value $u_{0}$,

$$
s\left(x_{0}, y_{0}, z_{0}\right)=0, \quad u\left(x_{0}, y_{0}, z_{0}\right)=u_{0} .
$$

3) The function $v(x, y, z)$ is infinitely many valued, its values corresponding to a given $(x, y, z)$ being all congruent modulo $2 \pi, b u t \cos v$ and $\sin v$ are single valued functions

$$
\cos v=a(x, y, z), \quad \sin v=b(x, y, z)
$$

of class $C^{\prime}$ in $L_{\delta}^{\prime}$.

4) Let $C$ be a curve

$$
x=f(t), \quad y=g(t), \quad z=h(t)
$$$$
(0 \leqq t \leqq T),
$$

with a continuously turning tangent, which intersects $\mathfrak{L}$ at a point $\boldsymbol{P}$ $\left(x_{0}, y_{0}, z_{0}, u_{0}\right)$ for $t=0$, but is not tangent to $\mathfrak{L}$ at $P$, and for which $t$ is the length of arc. The tangent to $C$ at $P$ determines uniquely $a$ direction

$$
l \cos v_{0}+\lambda \sin v_{0},
$$$$
m \cos v_{0}+\mu \sin v_{0},
$$$$
n \cos v_{0}+\nu \sin v_{0},
$$

coplanar with the tangents to $C$ and $\mathfrak{Q}$ at $P$, and lying on the same side of the tangent to $\mathbb{Q}$ with the positive tangent to $C$. As $t$ approaches zero the functions $s(t), u(t), a(t), b(t)$ defined by substituting the values of $x, y, z$ 
from $(C)$ in the functions (12) and (13), approach the limits $0, u_{0}, \cos v_{0}$, $\sin v_{0}$, and the functions $s(t), u(t)$ have derivatives at $t=0$ which have the values

$$
s^{\prime}(0)=\sin \omega \csc \theta, \quad u^{\prime}(\theta)=\cos \omega-\sin \omega \cot \theta,
$$

where $\omega$ is the angle between the positive tangents to $C$ and $\mathfrak{L}$, and $\theta$ is the corresponding angle for $E$ and $\mathbb{Q}$.

The properties 1) and 3) follow readily from the usual theorems concerning implicit functions and the periodicity of $\phi, \psi, \chi$ with respect to $v$. For at any solution $\left(x^{\prime}, y^{\prime}, z^{\prime}: s^{\prime}, u^{\prime}, v^{\prime}\right)$ of equations $(E)$ for which $\left(x^{\prime}, y^{\prime}, z^{\prime}\right)$ is in $\mathfrak{L}_{\delta}^{\prime}$ and $\left(s^{\prime}, u^{\prime}, v^{\prime}\right)$ is in $\varsigma_{\epsilon}^{\prime}$, the functional determinant of $\phi, \psi, \chi$ with respect to $s, u, v$ is different from zero.

If $P_{0}$ is a point $\left(x_{0}, y_{0}, z_{0}\right)$ on $\mathfrak{Q}$ corresponding to the value $u_{0}$, then for any $\eta>0$ a value $\rho>0$ can be selected so that $|s(x, y, z)|<\eta$ whenever $(x, y, z)$ is in the $\rho$-neighborhood of $P_{0}$. For the values $(s, u, v)$ in $\mathfrak{S}_{\mathrm{e}}^{\prime}$ for which $s \geqq \eta$ define a closed set of points $(x, y, z)$ distinct from $P_{0}$, whose minimum distance from the point $P_{0}$ is a positive constant $\rho \sqrt{3}$. Hence any $(x, y, z)$ in the $\rho$-neighborhood of $P_{0}$ will have its distance from $P_{0}$ less than $\rho$, and will necessarily correspond to a value of $s$ for which $0<s<\eta$. In a similar manner the points of $\mathfrak{S}_{\mathrm{e}}$ for which $\left|u-u_{0}\right| \geqq \eta$ define a closed set of $(x, y, z)$ points distinct from $P_{0}$, and the continuity of $u(x, y, z)$ as stated in 2) can be proved.

It remains to consider the property 4). An application of Taylor's formula shows that the equations

$$
\begin{aligned}
& \frac{1}{t}\left\{\phi\left(\sigma t, u_{0}+\tau t, v\right)-x_{0}\right\}=\frac{f(t)-x_{0}}{t}, \\
& \frac{1}{t}\left\{\psi\left(\sigma t, u_{0}+\tau t, v\right)-y_{0}\right\}=\frac{g(t)-y_{0}}{t}, \\
& \frac{1}{t}\left\{\chi\left(\sigma t, u_{0}+\tau t, v\right)-z_{0}\right\}=\frac{h(t)-z_{0}}{t},
\end{aligned}
$$

reduce for $t=0$ to

$$
\sigma \phi_{.}+\tau \alpha=f^{\prime}(0), \quad \sigma \psi_{\text {. }}+\tau \beta=g^{\prime}(0), \quad \sigma \chi_{\text {。 }}+\tau \gamma=h^{\prime}(0),
$$

where $\sigma \geqq 0$ and the arguments of $\phi_{s}, \psi_{s}, \chi_{t}$ are $\left(0, u_{0}, v\right)$. As a result of the statements proved on page 334 , there can be but one value $v_{0}$ (modulo $2 \pi$ ) for which these equations are satisfied, and the corresponding direction (14) has the properties described above. It follows that equations (15) have for $t=0$ unique solutions

$$
\sigma_{0}=\sin \omega \csc \theta, \quad \tau_{0}=\cos \omega-\sin \omega \cot \theta, \quad v_{0}
$$


determined by equations (16). The functional determinant of equations (15) for the values $\left(\sigma_{0}, \tau_{0}, v_{0}, t=0\right)$ is found with the help of the for:nula (10) to be the determinant (6). Hence these equations have continuous solutions $\sigma(t)$, $\tau(t), v(t)$ reducing to $\sigma_{0}, \tau_{0}, v_{0}$ for $t=0$. The corresponding functions

$$
s=\sigma t, \quad u=u_{0}+\tau t, \quad \cos v=a(t), \quad \sin v=b(t)
$$

define solutions of equations $(E)$, and hence must coincide with the functions $s(t), u(t), a(t), b(t)$ determined under 4). It follows readily that $s^{\prime}(0), u^{\prime}(0)$ exist and have the values $\sigma_{0}, \tau_{0}$, while $a(t), b(t)$, approach $\cos v_{0}$ and $\sin v_{0}$, respectively, as $t$ approaches zero. 